TITLE:

\title{
Error map construction for rotary axes on five-axis machine tools by on-the-machine measurement using a touch-trigger probe
}

\author{
$\operatorname{AUTHOR}(S)$ : \\ Ibaraki, Soichi; Iritani, Takeyuki; Matsushita, \\ Tetsuya
}

\section{CITATION:}

Ibaraki, Soichi ... [et al]. Error map construction for rotary axes on five-axis machine tools by on-the-machine measurement using a touch-trigger probe. International Journal of Machine Tools and Manufacture 2013, 68: 21-29

ISSUE DATE:

2013-05

URL:

http://hdl.handle.net/2433/172233

\section{RIGHT:}

(c) 2013 Elsevier Ltd.; この論文は出版社版でありません。引用の際には 出版社版をご確認ご利用ください。; This is not the published version. Please cite only the published version. 


\title{
Error map construction for rotary axes on five-axis machine tools by on-the-machine measurement using a touch-trigger probe
}

\author{
Soichi Ibaraki ${ }^{\mathrm{a}}$, Takeyuki Iritani ${ }^{\mathrm{a}}$, Tetsuya Matsushita ${ }^{\mathrm{b}}$ \\ ${ }^{a}$ Department of Micro Engineering, Kyoto University, \\ Yoshida-honmachi, Sakyo-ku, Kyoto 606-8501, Japan. Phone/Fax: +81-75-753-5227 \\ ${ }^{b}$ Okuma Corporation, Oguchi-cho, Niwa-gun, Aichi 480-0193, Japan.
}

\begin{abstract}
Position-dependent geometric errors, or "error map," of a rotary axis represent how position and orientation of the axis of rotation change with its rotation. This paper proposes a scheme to calibrate the error map of rotary axes by on-the-machine measurement of test pieces by using a contact-type touch-trigger probe installed on the machine's spindle. The present scheme enables more efficient and automated error calibration, which is crucial to implement periodic check of rotary axes error map or periodic update of its numerical compensation for five-axis machine tools. The uncertainty analysis of the error calibration is also presented with a particular interest in the influence of error motions of linear axes. The experimental demonstration is presented.
\end{abstract}

Keywords: Five-axis machine tools, rotary axis, error map, location errors, touch-trigger probe, error calibration

Email address: ibaraki@prec.kyoto-u.ac.jp (Soichi Ibaraki) 


\section{Introduction}

Machine tools with two rotary axes to tilt and rotate a tool and/or a workpiece, in addition to three orthogonal linear axes, are collectively called five-axis machine tools. Many error calibration schemes for five-axis machine tools have been recently studied as is reviewed in $[1,2]$.

In ISO 230-7 [3], location errors of a rotary axis represent position and orientation errors of the axis average line of a rotary axis, i.e. the straight line representing the mean location and orientation of its axis of rotation. Location errors are clearly one of the most fundamental error factors in the five-axis kinematics. ISO 10791-1 [4], currently under a revision process in ISO TC39/SC2, contains quasi-static or no-load tests with a main interest in calibrating location errors of rotary axes. The application of the ball bar measurement to the calibration of location errors have been reported in many research works $[5,6,7]$. The R-test $[8,9,10,11]$ can be seen its extension to three-dimensional measurement. Dynamic interpolation tests using the ball bar or the R-test are included in ISO/DIS 10791-6:2012 [12], also currently under a revision process in ISO TC39/SC2.

It must be emphasized that location errors only represent "average" positions or orientations of a rotary axis. The position and the orientation of the axis of rotation may change with its rotation. It is of a practical importance to calibrate not only the "average" of error motions, but also how error motion changes with its rotation. For example, for the machine configuration with a rotary table ( $\mathrm{C}$-axis) mounted on a swiveling axis (A-axis) (see Fig. 1), C-axis error motions may be larger when the rotary table is vertical (at $A= \pm 90^{\circ}$ ) due to e.g. the gravity-induced deformation of its bearings. Such an influence of the angular position of a rotary axis on its error motions, or error motions of the other axis mounted on it, can be parameterized by location errors that vary depending on the angular position of a rotary axis. Such position-dependent geometric errors [13], or an "error map" of a rotary axis [2] models a larger class of more complex error motions of rotary axis, such as the gravity deformation, angular positioning error, pure radial error motions or tilt error motions of a rotary axis. The application of the R-test to numerically calibrate such an "error map" was presented by a part of the authors $[10,14]$.

Many machine tools in today's market have on-machine probing capability, usually used for part setup compensation. High-accuracy touch-trigger probes for machine tools, which typically have one-directional measurement 
repeatability less than $1 \mu \mathrm{m}$, are available from some vendors. The standard of test codes for the performance of such a touch-trigger probe is available (ISO 230-10 [15]). By its nature, such a probe has good communication capability with a CNC system, which potentially facilitates the automation of error calibration and compensation. For rotary axes, probe-based calibration of their axis average line position can be done on some commercial CNCs [16]. Its extension to a set of all location errors of rotary axes has been reported in the literature $[17,18]$. The authors also presented, in our previous publication [19], a probe-based calibration scheme for location errors. ISO 10360-3:2000 [20] describes a similar test for coordinate measuring machines (CMMs) with a rotary table as the fourth axis.

While all these past works focused only on the calibration of location errors, the objective of this paper is to present a error calibration scheme based on on-the-machine measurement by a touch-trigger probe to identify not only location errors, but also position-dependent geometric errors. Its experimental demonstration on a commercial five-axis machining center of a tilting rotary table configuration will be presented.

\section{Error parameters to be identified and measuring instrument}

\subsection{Machine configuration}

This paper considers a 5 -axis machine configuration with a titling rotary table (driven by A- and C-axes) depicted in Fig. 1. It must be emphasized that the basic idea of this paper can be straightforwardly extended to any configurations of five-axis machines.

\subsection{Geometric error parameters to be identified}

Table 1 shows position-dependent geometric errors for the machine configuration in Fig. 1. It is to be noted that parameters associated with A-axis are dependent only on the A-axis angular position, while those associated with $\mathrm{C}$-axis are dependent on both $\mathrm{A}$ - and $\mathrm{C}$-axis angular positions. This is because an error motion of C-axis may be affected by $\mathrm{A}$-axis angular position (its typical causes include gravity-induced deformation of bearings or mechanical structure). 
To eliminate the redundancy, geometric errors of C-axes are defined such that:

$$
\begin{aligned}
\sum_{j=1}^{N_{c}} \delta x_{C A}\left(A_{i}, C_{j}\right) & =\sum_{j=1}^{N_{c}} \delta y_{C A}\left(A_{i}, C_{j}\right)=\sum_{j=1}^{N_{c}} \delta z_{C A}\left(A_{i}, C_{j}\right)=0 \\
\sum_{j=1}^{N_{c}} \alpha_{C A}\left(A_{i}, C_{j}\right) & =\sum_{j=1}^{N_{c}} \beta_{C A}\left(A_{i}, C_{j}\right)=\sum_{j=1}^{N_{c}} \gamma_{C A}\left(A_{i}, C_{j}\right)=0
\end{aligned}
$$

It is important to note that this paper assumes geometric errors of linear axes (X, Y, and Z-axes) are negligibly small compared to those of rotary axes. As was reviewed in Section 1, many five-axis error calibration methodologies have been recently studied (e.g. ball bar tests and the R-test). All of them only measure the relative displacement of the spindle tip to the table, and it is therefore not possible in principle to separate error motions of rotary axes and linear axes. To identify error motions of rotary axes, error motions of linear axes are required to be separately pre-calibrated by conventional measurement (e.g. ISO 10791-1 [4]). The influence of linear axis error motions on the estimation uncertainty will be discussed in Section 6 .

\subsection{Kinematic Modeling of Five-axis Machine}

The kinematic model to compute the tool center position (TCP) relative to the work table is the basis of the error calibration presented in this paper. Since its derivation can be found in many previous publications $[6,10,22]$, this subsection only briefly reviews it.

Define the machine coordinate system as the coordinate system fixed to the $\mathrm{Y}$-axis frame. This coordinate system is independent of A- and Crotations. Suppose that the TCP in the machine coordinate system is given by ${ }^{r} q \in \mathbb{R}^{3}$. The left-side superscript $r$ represents a vector in the machine coordinate system.

Define the workpiece coordinate system as the coordinate system with its Z-axis attached to the machine's C-axis of rotation, and with its origin at the Z-height where the A-axis of rotation intersects with its $\mathrm{YZ}$ plane. The homogeneous transformation matrix (HTM) representing the transformation from the workpiece coordinate system to the machine coordinate system is 
given by:

$$
\begin{aligned}
{ }^{r} T_{w}= & { }^{y} T_{a}{ }^{a} T_{c} \\
{ }^{a} T_{c}= & D_{x}\left(\delta x_{C A}\left(A_{i}, C_{j}\right)\right) D_{y}\left(\delta y_{C A}\left(A_{i}, C_{j}\right)\right) D_{z}\left(\delta z_{C A}\left(A_{i}, C_{j}\right)\right) \\
& D_{a}\left(\alpha_{C A}\left(A_{i}, C_{j}\right)\right) D_{b}\left(\beta_{C A}\left(A_{i}, C_{j}\right)\right) D_{c}\left(\gamma_{C A}\left(A_{i}, C_{j}\right)\right) D_{c}\left(-C_{j}\right) \\
{ }^{y} T_{a}= & D_{x}\left(\delta x_{A Y}\left(A_{i}\right)\right) D_{y}\left(\delta y_{A Y}\left(A_{i}\right)\right) D_{z}\left(\delta z_{A Y}\left(A_{i}\right)\right) \\
& D_{a}\left(\alpha_{A Y}\left(A_{i}\right)\right) D_{b}\left(\beta_{A Y}\left(A_{i}\right)\right) D_{c}\left(\gamma_{A Y}\left(A_{i}\right)\right) D_{a}\left(-A_{i}\right)
\end{aligned}
$$

where $D_{x}(x), D_{y}(y)$, and $D_{z}(z) \in \mathbb{R}^{4 \times 4}$ represent the HTM for linear motions in X-, Y-, and Z-directions. $D_{a}(a), D_{b}(b)$, and $D_{c}(c) \in \mathbb{R}^{4 \times 4}$ represent the HTM for angular motions about $X, Y$ and $Z$ axes. See e.g. [6, 22] for their formulation. $A_{i}$ and $C_{j} \in \mathbb{R}$ represent the command angular position of A and $\mathrm{C}$ axes, respectively.

Hence, the TCP in the workpiece coordinate system, ${ }^{w} q \in \mathbb{R}^{3}$, is given by:

$$
\left[\begin{array}{c}
{ }^{w} q \\
1
\end{array}\right]=\left({ }^{r} T_{w}\right)^{-1} \cdot\left[\begin{array}{c}
{ }^{r} q \\
1
\end{array}\right]
$$

The left-side superscript $w$ denotes the vector defined in the workpiece coordinate system.

\subsection{Measuring Instrument}

This paper uses a typical contact-type touch-trigger probe for discretepoint probing. The probe approaches to the object surface in the direction normal to it. When the contact of a probe ball with the object is detected, a signal is sent to a CNC to stop the drive and record its position in the machine coordinate system. The position of the contact point on the surface is calculated from the machine position, the approaching direction, the calibrated ball radius, and the probe's pre-travel. The term "probed point" hereafter represents the measured position of the contact point on the test piece surface. According to the probe software's standard procedure or [15], the pre-travel variation for different approaching directions must be compensated.

\section{Probing procedure}

The proposed test procedure is described as follows: 
1. Three test pieces of a square column geometry are fixed on the machine's rotary table as shown in Fig. 2(a). The nominal position and size of each test piece is also shown in Fig. 2(a). Each test piece is aligned to the machine's linear axes only roughly.

2. At (I) $A_{i}=0^{\circ}$ and $C_{j}=0^{\circ}$, total 15 points are probed from the direction normal to the surface, as illustrated in Fig. 2(a). In Fig. 2(a), the index $k$ labels the probing sequence.

3. Then, index A- and C-axes at the given set of angular positions; in our experiment, each combination of $A_{i}=0,-30,-60,-90^{\circ}\left(N_{a}=4\right)$ and $C_{j}=0,60,120,180,240,300^{\circ}\left(N_{c}=6\right)$. The probing sequence is performed at total $N_{a} \cdot N_{c}=24$ positions.

4. The number of probed points may vary at each indexed angle. For example, at $A_{i}=0^{\circ}$ and $C_{j}=60^{\circ}$, total 12 points are probed as illustrated in Fig. 2(b). Same points are probed at $A_{i}=0^{\circ}$ and $C_{j}=$ $60, \cdots, 300^{\circ}$.

5. At $A_{i} \neq 0^{\circ}$, to avoid unwanted interference of the probe to test pieces, less points are probed. For example, Fig. 2(c) shows probed points at $A_{i}=-90^{\circ}$ and $C_{j}=0^{\circ}$. Total 8 points are probed.

For indexed angles $A_{i}$ and $C_{j}$, suppose that the reference location of the $k$-th probed point is represented by $\tilde{p}(i, j, k) \in \mathbb{R}^{3}$. Denote its measured position by $p(i, j, k) \in \mathbb{R}^{3}$.

Remark \#1:

Our previous work [19] presented the identification of location errors of rotary axes by probing a single test piece. In this paper, multiple test pieces are used to magnify the influence of angular (tilt) error motions of rotary axis in measured displacements. For example, as illustrated in Fig. 3, the angular positioning error of C-axis, represented by $\gamma_{C A}\left(A_{i}, C_{j}\right)$, affects the Y-position of probed points by:

$$
y_{2}-y_{1}=L_{1} \cdot \gamma_{C A}\left(C_{j}, A_{i}\right)
$$

where $y_{1}$ and $y_{2}$ represent the Y-position of each probed point. The influence of the measurement uncertainty in $y_{1}$ and $y_{2}$ to the identification of $\gamma_{C \wedge}\left(C_{j}, A_{i}\right)$ becomes smaller when $L_{1}$ is larger.

When the test objective is to identify location errors only, the "average" orientation of $\mathrm{C}$-axis over $360^{\circ}$ rotation can be observed by measuring the trajectory of the position of a single test piece. On the other hands, for the identification of position-dependent geometric errors, the orientation of 
$\mathrm{C}$-axis must be measured at each indexed angle, $C_{j}$. By observing the trajectory of a single point in the workpiece coordinate system (as in the R-test or the ball bar test), it is in principle not possible to observe the tilt error motion of the rotary table at each indexed angle. The observability of tilt error motion of both $\mathrm{C}$ - and $\mathrm{A}$-axes is an advantage of the present probe-based approach, although it is restricted to static measurement. Considering the symmetry of test piece locations in both $\mathrm{X}$ - and $\mathrm{Y}$-directions, we used three test pieces as shown in Fig. 2.

Remark \#2:

The present probing procedure can be modified according to experimental setup. For example, the present procedure is for the machines where the A-axis rotates only from $A_{i}=0^{\circ}$ to $90^{\circ}$. When the A-axis rotates e.g. from $A_{i}=-180^{\circ}$ to $180^{\circ}$, the probing sequence should be modified in an analogous manner.

\section{Algorithm to identify position-dependent geometric errors}

\subsection{Calculation of table position and orientation}

The objective of the present algorithm is to identify position-dependent geometric errors of $\mathrm{A}$ - and C-axes shown in Table 1 for each of $A_{i}$ and $C_{j}$ $\left(i=1 \cdots N_{a}, j=1 \cdots N_{c}\right)$ from probed positions, $p(i, j, k)\left(k=1 \cdots N_{k}\right)$.

For $\left(A_{i}, C_{j}\right)$, represent the position error of the workpiece coordinate system (attached to the rotary table) from its nominal position in $\mathrm{X}, \mathrm{Y}$, and $\mathrm{Z}$ directions of the machine coordinate system by $(\Delta x(i, j), \Delta y(i, j), \Delta z(i, j))$. Represent its orientation error around $X, Y$, and $Z$ directions of the machine coordinate system by $(\Delta a(i, j), \Delta b(i, j), \Delta c(i, j))$. They can be roughly seen as position and orientation errors of the rotary table at $A_{i}$ and $C_{j}$.

As the first step of the proposed algorithm, this subsection presents an algorithm to calculate $\Delta x(i, j), \cdots, \Delta c(i, j)$ from probed positions, $p(i, j, k)$.

The $k$-th probed point at $A_{i}=C_{j}=0^{\circ}, p(1,1, k)\left(k=1 \cdots N_{k}\right)$, nominally moves at given $A_{i}$ and $C_{j}$ to:

$$
\left[\begin{array}{c}
\tilde{p}(i, j, k) \\
1
\end{array}\right]=D_{a}\left(-A_{i}\right) D_{c}\left(-C_{j}\right)\left[\begin{array}{c}
p(1,1, k) \\
1
\end{array}\right]
$$


When there exist table position and orientation errors at $A_{i}$ and $C_{j}, \Delta x(i, j), \cdots, \Delta \gamma(i, j)$, it moves to:

$$
\begin{aligned}
{\left[\begin{array}{c}
\hat{p}(i, j, k) \\
1
\end{array}\right]=} & D_{x}(\Delta x(i, j)) D_{y}(\Delta y(i, j)) D_{z}(\Delta z(i, j)) \cdot \\
& D_{a}(\Delta a(i, j)) D_{b}(\Delta b(i, j)) D_{c}(\Delta c(i, j))\left[\begin{array}{c}
\tilde{p}(i, j, k) \\
1
\end{array}\right]
\end{aligned}
$$

It must be noted that a touch-trigger probe is sensitive to the displacement of the probed point only in its approaching direction (for example, when the probe approaches to the test piece surface in the X-direction, Y- and Zpositions of the probed point cannot be observed). For the $k$-th point, suppose that the approaching direction is given by a unit vector, $\tilde{n}(i, j, k) \in \mathbb{R}^{3}$. Then, the table position and orientation at $A_{i}$ and $C_{j}, \Delta x(i, j), \cdots, \Delta c(i, j)$, are computed by solving the following minimization problem at each $(i . j)$ :

$$
\min _{\Delta x(i, j), \cdots, \Delta \gamma(i, j)} \sum_{k=1}^{N_{k}}\{\Delta p(i, j, k) \cdot \tilde{n}(i, j, k)\}^{2}
$$

where:

$$
\Delta p(i, j, k)=p(i, j, k)-\hat{p}(i, j, k)
$$

represents the displacement of the probed point, measured at $\left(A_{i}, C_{j}\right)$, from its initial position, measured at $A_{i}=C_{j}=0^{\circ}$.

When $\Delta x, \Delta y, \Delta z, \Delta a, \Delta b$, and $\Delta c \in \mathbb{R}$ are sufficiently small, the following approximation generally holds:

$D_{x}(\Delta x) D_{y}(\Delta y) D_{z}(\Delta z) D_{a}(\Delta a) D_{b}(\Delta b) D_{c}(\Delta c) \approx\left[\begin{array}{cccc}1 & -\Delta c & \Delta b & \Delta x \\ \Delta c & 1 & -\Delta a & \Delta y \\ -\Delta b & \Delta a & 1 & \Delta z \\ 0 & 0 & 0 & 1\end{array}\right]$ 
By using this, the problem (7) can be approximated as a linear programming problem by:

$$
\min _{\Delta x(i, j) \sim \Delta \gamma(i, j)} \sum_{k=1}^{N_{k}}[\{p(i, j, k)-\tilde{p}(i, j, k)
$$

$\left.\left.-\left[\begin{array}{cccccc}1 & 0 & 0 & 0 & \tilde{p}_{z}(i, j, k) & -\tilde{p}_{y}(i, j, k) \\ 0 & 1 & 0 & -\tilde{p}_{z}(i, j, k) & 0 & \tilde{p}_{x}(i, j, k) \\ 0 & 0 & 1 & \tilde{p}_{y}(i, j, k) & -\tilde{p}_{x}(i, j, k) & 0\end{array}\right]\left[\begin{array}{c}\Delta x(i, j) \\ \Delta y(i, j) \\ \Delta z(i, j) \\ \Delta a(i, j) \\ \Delta b(i, j) \\ \Delta c(i, j)\end{array}\right]\right\} \cdot \tilde{n}(i, j, k)\right]^{2}$

where $\tilde{p}(i, j, k)=\left[\tilde{p}_{x}(i, j, k), \tilde{p}_{y}(i, j, k), \tilde{p}_{z}(i, j, k)\right]^{T} \in \mathbb{R}^{3}$. This can be solved by the least square method.

\subsection{Calculation of position-dependent geometric errors of rotary axes}

The table position and orientation at $A_{j}$ and $C_{i}, \Delta x(i, j), \cdots, \Delta c(i, j)$, are then separated into position-dependent geometric errors of $\mathrm{A}$ - and $\mathrm{C}$-axes shown in Table 1.

When the nominal TCP in the machine coordinate system is given by ${ }^{r} \tilde{p} \in \mathbb{R}^{3}$, its actual position under position-dependent geometric errors of Aand C-axes, ${ }^{r} p \in \mathbb{R}^{3}$, is given based on the kinematic model (3) as follows:

$$
\left[\begin{array}{c}
{ }^{r} p \\
1
\end{array}\right]={ }^{r} T_{w}\left({ }^{r} \tilde{T}_{w}\right)^{-1} \cdot\left[\begin{array}{c}
{ }^{r} \tilde{p} \\
1
\end{array}\right]
$$

where ${ }^{r} T_{w} \in \mathbb{R}^{4 \times 4}$ is given by Eq. (2). ${ }^{r} \tilde{T}_{w} \in \mathbb{R}^{4 \times 4}$ is the HTM representing the nominal rotation by $A_{i}$ and $C_{j}$, i.e.

$$
{ }^{r} \tilde{T}_{w}=D_{a}\left(-A_{i}\right) D_{c}\left(-C_{j}\right)
$$

From this formulation, with the approximation (9), the relationship of the table position and orientation, $\Delta x(i, j), \cdots, \Delta c(i, j)$, and position-dependent geometric errors of $\mathrm{A}$ - and $\mathrm{C}$-axes given in Table 1 is formulated as fol- 
lows $[10]$ :

$$
\begin{aligned}
\Delta x(i, j) & =\delta x_{A Y}\left(A_{i}\right)+\delta x_{C A}\left(A_{i}, C_{j}\right)-\Delta x_{0}(i . j) \\
\Delta y(i, j) & =\delta y_{A Y}\left(A_{i}\right)+\delta y_{C A}\left(A_{i}, C_{j}\right) \cos A_{i}+\delta z_{C A}\left(A_{i}, C_{j}\right) \sin A_{i}-\Delta y_{0}(i . j) \\
\Delta z(i, j) & =\delta z_{A Y}\left(A_{i}\right)+\delta z_{C A}\left(A_{i}, C_{j}\right) \cos A_{i}-\delta y_{C A}\left(A_{i}, C_{j}\right) \sin A_{i}-\Delta z_{0}(i . j) \\
\Delta a(i, j) & =\alpha_{A Y}\left(A_{i}\right)+\alpha_{C A}\left(A_{i}, C_{j}\right)-\Delta a_{0}(i . j) \\
\Delta b(i, j) & =\beta_{A Y}\left(A_{i}\right)+\beta_{C A}\left(A_{i}, C_{j}\right) \cos A_{i}+\gamma_{C A}\left(A_{i}, C_{j}\right) \sin A_{i}-\Delta b_{0}(i . j) \\
\Delta c(i, j) & =\gamma_{A Y}\left(A_{i}\right)+\gamma_{C A}\left(A_{i}, C_{j}\right) \cos A_{i}-\beta_{C A}\left(A_{i}, C_{j}\right) \sin A_{i}-\Delta c_{0}(i . j)
\end{aligned}
$$

Recall that the table position and orientation, $\Delta x(i, j), \cdots, \Delta c(i, j)$, are defined relative to those at $A_{i}=C_{j}=0^{\circ}(i=j=1)$ (see Eq. (8)). In other words, $\Delta x(i, j), \cdots, \Delta c(i, j)$ are restricted at $i=j=1$ as:

$$
\Delta x(1,1)=\cdots=\Delta c(1,1)=0
$$

In Eq. (13), the terms $\Delta x_{0}(i . j), \cdots \Delta c_{0}(i . j)$ represent this restriction. From Eq. (5), they are given by:

$$
\begin{aligned}
& {\left[\begin{array}{c}
\Delta x_{0}(i, j) \\
\Delta y_{0}(i, j) \\
\Delta z_{0}(i, j) \\
1
\end{array}\right]=D_{a}\left(-A_{i}\right) D_{c}\left(-C_{j}\right)\left[\begin{array}{c}
\delta x_{C A}\left(A_{1} \cdot C_{1}\right) \\
\delta y_{A Y}\left(A_{1}\right)+\delta y_{C A}\left(A_{1}, C_{1}\right) \\
\delta z_{A Y}\left(A_{1}\right) \\
1
\end{array}\right]} \\
& {\left[\begin{array}{c}
\Delta a_{0}(i, j) \\
\Delta b_{0}(i, j) \\
\Delta c_{0}(i, j) \\
1
\end{array}\right]=D_{a}\left(-A_{i}\right) D_{c}\left(-C_{j}\right)\left[\begin{array}{c}
\alpha_{C A}\left(A_{1} \cdot C_{1}\right) \\
\beta_{A Y}\left(A_{1}\right)+\beta_{C A}\left(A_{1}, C_{1}\right) \\
\gamma_{A Y}\left(A_{1}\right) \\
1
\end{array}\right]}
\end{aligned}
$$

where the following initial condition is imposed to eliminate the redundancy in the definition of geometric error parameters of $\mathrm{A}$ - and C-axes:

$$
\delta x_{A Y}\left(A_{1}\right)=\delta z_{C A}\left(A_{1}, C_{1}\right)=\alpha_{A Y}\left(A_{1}\right)=\gamma_{C A}\left(A_{1}, C_{1}\right)=0
$$


From Eq. (13), under the definition in Eq. (1), geometric errors of A-axis can be computed from $\Delta x(i, j), \cdots, \Delta c(i, j)$ by:

$$
\begin{aligned}
\delta \hat{x}_{A Y}\left(A_{i}\right) & =\operatorname{mean}_{j}\left\{\Delta x(i, j)+\Delta x_{0}(i . j)\right\} \\
\delta \hat{y}_{A Y}\left(A_{i}\right) & =\operatorname{mean}_{j}\left\{\Delta y(i, j)+\Delta y_{0}(i . j)\right\} \\
\delta \hat{z}_{A Y}\left(A_{i}\right) & =\operatorname{mean}_{j}\left\{\Delta z(i, j)+\Delta z_{0}(i . j)\right\} \\
\hat{\alpha}_{A Y}\left(A_{i}\right) & =\operatorname{mean}_{j}\left\{\Delta a(i, j)+\Delta a_{0}(i . j)\right\} \\
\hat{\beta}_{A Y}\left(A_{i}\right) & =\operatorname{mean}_{j}\left\{\Delta b(i, j)+\Delta b_{0}(i . j)\right\} \\
\hat{\gamma}_{A Y}\left(A_{i}\right) & =\operatorname{mean}_{j}\left\{\Delta c(i, j)+\Delta c_{0}(i . j)\right\}
\end{aligned}
$$

Then, by solving Eq.(13), geometric errors of C-axis are computed by:

$$
\begin{gathered}
{\left[\begin{array}{c}
\Delta \hat{x}_{C A}\left(A_{i}, C_{j}\right) \\
\Delta \hat{y}_{C A}\left(A_{i}, C_{j}\right) \\
\Delta \hat{z}_{C A}\left(A_{i}, C_{j}\right) \\
1
\end{array}\right]=\left\{D_{a}\left(-A_{i}\right)\right\}^{-1}\left[\begin{array}{c}
\Delta x(i, j)+\Delta x_{0}(i, j)-\delta x_{A Y}\left(A_{i}\right) \\
\Delta y(i, j)+\Delta y_{0}(i, j)-\delta y_{A Y}\left(A_{i}\right) \\
\Delta z(i, j)+\Delta z_{0}(i, j)-\delta z_{A Y}\left(A_{i}\right) \\
1
\end{array}\right]} \\
{\left[\begin{array}{c}
\hat{\alpha}_{C A}\left(A_{i}, C_{j}\right) \\
\hat{\beta}_{C A}\left(A_{i}, C_{j}\right) \\
\hat{\gamma}_{C A}\left(A_{i}, C_{j}\right) \\
1
\end{array}\right]=\left\{D_{a}\left(-A_{i}\right)\right\}^{-1}\left[\begin{array}{c}
\Delta a(i, j)+\Delta a_{0}(i, j)-\alpha_{A Y}\left(A_{i}\right) \\
\Delta b(i, j)+\Delta b_{0}(i, j)-\beta_{A Y}\left(A_{i}\right) \\
\Delta c(i, j)+\Delta c_{0}(i, j)-\gamma_{A Y}\left(A_{i}\right) \\
1
\end{array}\right]}
\end{gathered}
$$

\section{Experimental case study}

\subsection{Experimental setup}

The present error calibration scheme is applied to a commercial middlesize five-axis machining center of the configuration shown in Fig. 1. Its major specifications are shown in Table 2. A touch-trigger probe, RMP-600 by Renishaw, is used in experiments. RMP-600 employs strain gauges to detect the contact of the probe ball. Its major specifications are shown in Table 3.

Three test pieces of carbon steel JIS S50C are used (approximate size: $50 \times 50 \times 150 \mathrm{~mm}$ ). Their setup on the machine table is shown in Fig. 2 . The probing procedure was presented in Section 3. Total 219 points were probed, and total measurement time was about $28 \mathrm{~min}$. Figure 4 shows the experimental setup. To check the repeatability of the measurement, the 
probing procedure was repeated for total four times.

\subsection{Measurement result}

Figure 5 shows graphical presentation of measured results. Only (a) $A_{i}=0^{\circ}, C_{j}=180^{\circ}$ and (b) $A_{i}=-90^{\circ}, C_{j}=0^{\circ}$ are shown as examples. In the plots, red dots represent nominal probed positions, $\tilde{p}(i, j, k)$ in Eq. (5). Black dots represent the measured displacement of each probed point, $p(i, j, k)$. The displacement is magnified 200 times. The line segment from the nominal point (red dot) directed normal to the test piece surface represents the probe's sensitive direction. The measured point (black dot) is always on this line.

At each $\left(A_{i}, C_{j}\right)$, the table position, $(\Delta x(i, j), \Delta y(i, j), \Delta z(i, j))$, and orientation, $(\Delta a(i, j), \Delta b(i, j), \Delta c(i, j))$, are calculated from measured positions as presented in Section 4.1. Boxes painted in green in Fig. 5 show the position and the orientation of each test piece calculated from $\Delta x(i, j), \cdots, \Delta c(i, j)$. Since side faces of test piece are measured only at the same height, their inclination around X- or Y-axes cannot be seen. In Fig. 5, all side faces of test piece are assumed to be vertical.

From graphical presentation shown in Fig. 5, many intuitive observations can be made on error motions of rotary axes. For example, Fig. 5(a-1) shows that test pieces are shifted by about $40 \mu \mathrm{m}$ in the X-direction, and by about $50 \mu \mathrm{m}$ in the $\mathrm{Y}$-direction with the $\mathrm{C}$-axis rotation from $C_{j}=0^{\circ}$ to $C_{j}=180^{\circ}$ at $A_{i}=0^{\circ}$. This is mostly caused by the miscalibration of the $\mathrm{C}$-axis average line position, represented by $\delta x_{A Y}\left(A_{i}\right)$ and $\delta y_{A Y}\left(A_{i}\right)$ at $A_{i}=0^{\circ}$. It can be furthermore observed in Fig. 5(b-1) that two lower test pieces are slightly shifted (by about $15 \mu \mathrm{m}$ ) to -Y direction compared the upper test piece at $A_{i}=90^{\circ}$. This suggests the angular positioning error of A-axis at $A_{i}=-90^{\circ}$.

\subsection{Identification of position-dependent geometric errors}

By the algorithm presented in Section 4.2, position-dependent geometric errors can be identified. Figures 6(a) to (f) show A-axis geometric errors, $\delta x_{A Y}\left(A_{i}\right), \cdots, \gamma_{A Y}\left(A_{i}\right)$. They represent how error motions of A-axis varies at $A_{i}=0,-30,-60$, and $-90^{\circ}$. Figure 7 shows position-dependent geometric errors of C-axis. Only those at $A_{i}=-90^{\circ}$ are shown as an example. To show the repeatability of the tests, estimates in four repeated tests are all 
shown. Error bars represent the estimation uncertainty due to contributors shown in Table 4 (see Section 6). The following observations can be made:

- A constant offset in $\delta x_{A Y}\left(A_{i}\right), \delta y_{A Y}\left(A_{i}\right)$, and $\delta z_{A Y}\left(A_{i}\right)$ (in Fig. 6(a) to (c)) represent the position error of the axis average line of A-axis (or C-axis), i.e. location errors, $\delta x_{A Y}^{0}, \delta y_{A Y}^{0}$, and $\delta z_{A Y}^{0}$.

- Not only these "average" position errors, but also gradual shift of C-axis centerline to the X-direction with A-axis rotation $\left(\Delta x_{A Y}\left(A_{i}\right)\right.$, Fig. 6(a)) and that of A-axis centerline to the Y-direction $\left(\Delta y_{A Y}\left(A_{i}\right)\right.$, Fig. 6(b)), are also observed (about $10 \mu \mathrm{m}$ for $A_{i}=0$ to $-90^{\circ}$ ). Such an error cannot be observed by evaluating location errors only.

- $\alpha_{A Y}\left(A_{i}\right)$ (in Fig. 6(d)) represents the angular positioning error of Aaxis. It is sufficiently small at $A_{i}=0^{\circ}$, but it becomes about $32 \mu \mathrm{m} / \mathrm{m}$ at $A_{i}=-90^{\circ}$. This is also a position-dependent geometric error.

- $\beta_{A Y}\left(A_{i}\right)$ and $\gamma_{A Y}\left(A_{i}\right)$ (in Fig. 6(e) and (f)) represent tilt error motions of A-axis. The orientation of $\mathrm{A}$-axis changes with its rotation by about $10 \mu \mathrm{m} / \mathrm{m}$ around both $\mathrm{Y}$ - and Z-axes.

\section{Uncertainty analysis}

The probing patterns presented in Section 3 probe nominally same points on test pieces at each $A_{i}$ and $C_{j}$. The error parameter calibration presented in Section 4 is based on the measured displacement of each point from its initial position (measured at $A_{i}=C_{j}=0^{\circ}$ ) in the workpiece coordinate system. Therefore, the geometric inaccuracy of test pieces or its setup (alignment) error does not impose significant effect on the estimates of geometric error parameters. This is an important feature of the proposed approach. On the other hands, as was stated in Section 2.2, the present scheme assumes negligibly small geometric errors of linear axes. Error motions of linear axes may have significant influence on the estimates.

It is therefore practically important to assess the uncertainty in identified geometric error parameters due to, especially, error motions of linear axes. Statistical analysis based on the Monte Carlo simulation is common and well established in the measurement uncertainty assessment [24]. The uncertainty analysis presented in this section is analogous to the one presented in $[25,26]$, and similar analysis was presented in our previous study [19]. 
Principal contributors to the uncertainty are listed in Table 4. Associated with the probing; only the unidirectional repeatability (random measurement error) is taken into account; the measurement error caused by the directional pre-travel variation or other factors are neglected assuming proper pre-calibration and compensation [15]. The main focus of this analysis is to assess the influence of error motions of linear axes. Error motions of linear axes are modelled as follows, analogously as in [26]:

$$
\begin{aligned}
& E_{x x}(x)=E_{x x, l i n} \cdot x+\operatorname{interpolate}\left(X_{i}, E_{x x, \text { rand }}\left(X_{i}\right), x\right) \\
& E_{y x}(x)=\operatorname{interpolate}\left(X_{i}, E_{y x, \text { rand }}\left(X_{i}\right), x\right) \\
& E_{z x}(x)=\operatorname{interpolate}\left(X_{i}, E_{z x, \text { rand }}\left(X_{i}\right), x\right)
\end{aligned}
$$

where $E_{x x}(x), E_{y x}(x)$, and $E_{z x}(x)$ respectively represent positioning errors in $\mathrm{X}, \mathrm{Y}$, and $\mathrm{Z}$ directions of $\mathrm{X}$-axis at the nominal position, $x$. The function interpolate represents the linear interpolation of the function $E_{*}\left(X_{i}\right)$ at $x$. $X_{i}$ is given by $X_{i}=p_{\text {rand }} \cdot i$, where $p_{\text {rand }}$ is a random number uniformly distributed in $[20,50] \mathrm{mm}$. The squareness errors of linear axes are also taken into account. Error motions of all the linear axes are modelled analogously. Angular errors of linear axes are neglected, assurning small-sized machines.

The magnitude of each error is chosen randomly with the normal distribution given in Table 4 . These distributions are taken from comparative measurements, manufacturer specifications or tolerances in ISO standards. At each probing point, the machine's positioning error and the test piece's position and orientation are calculated, and error parameters are identified by exactly the same procedure presented in Section 4 . Test setups are the same as shown in Fig. 2. Monte Carlo simulations are used with 1,000 runs to assess the uncertainty in the estimates.

Figures 6 and 7 also show, by error bars, the standard deviation of estimation uncertainty for each geometric error parameter.

\section{Conclusion}

Position-dependent geometric errors, or an "error map" of rotary axes, represent how the rotation of a rotary axis influences its error motions, or error motions of the other axis mounted on it. This paper proposes a scheme to calibrate an "error map" of rotary axes by on-the-machine measurement of test pieces by using a contact-type touch-trigger probe installed on the machine's spindle. Compared to conventional calibration schemes described 
in ISO/DIS 10791-1:2012 [4] and ISO/DIS 10791-6:2012 [12], where a precision sphere and a linear displacement sensor(s) or the ball bar is used, the proposed approach is more suitable to efficient and automated calibration procedure of error map. It is therefore advantageous in the application to periodic check of error map, or periodic updating of its numerical compensation.

The geometric inaccuracy and the setup error of test pieces impose only negligibly small influence on the estimates. Therefore, there is no need to use a calibrated artefact to perform the present scheme. On the other hand, similarly as many other methodologies reported in the literature on the calibration of rotary axes (e.g. ball bar measurement and the R-test), the present scheme measures the relative displacement of the spindle to the table, and it is therefore not possible in principle to separate error motions of rotary axes and linear axes. The uncertainty analysis was presented to quantitatively assess the influence of error motions of linear axes on the estimates of geometric error parameters.

\section{References}

[1] S. Ibaraki, W.Knapp, Indirect Measurement of Volumetric Accuracy for Three-axis and Five-axis Machine Tools: A Review, Int ' l J. of Automation Technology, 6 (2) (2012).

[2] H. Schwenke, W. Knapp, H. Haitjema, A. Weckenmann, R. Schmitt, F. Delbressine, Geometric error measurement and compensation of machines - An update, CIRP Annals - Manufacturing Technology 57 (2) (2008) 560-575.

[3] ISO 230-7:2006, Test code for machine tools - Part 7: Geometric accuracy of axes of rotation, 2006.

[4] ISO/DIS 10791-1:2012, Test conditions for machining centres - Part 1: Geometric tests for machines with horizontal spindle (horizontal Z-axis), 2012.

[5] Y. Kakino, Y. Ihara, H. Sato, H. Otsubo, "A Study on the motion accuracy of $\mathrm{NC}$ machine tools (7th report) -Measurement of motion accuracy of $\bar{\nu}$-axis machine by DBB tests-," J. of Japan Society for Precision Engineering, 60-5, pp.718-723, 1994 (in Japanese). 
[6] Y. Abbaszaheh-Mir, J. R. R. Mayer, G. Clotier, C. Fortin, Theory and simulation for the identification of the link geometric errors for a fiveaxis machine tool using a telescoping magnetic ball-bar, International Journal of Production Research 40 (18) (2002) 4781-4797.

[7] M. Tsutsumi, A. Saito, Identification and compensation of systematic deviations particular to 5-axis machining centers, International Journal of Machine Tools and Manufacture 43 (2003) 771-780.

[8] S. Weikert, R-Test, a New Device for Accuracy Measurements on Five Axis Machine Tools, Annals of the CIRP 53 (1) (2004) 429-432.

[9] B. Bringmann, W. Knapp, Model-based 'Chase-the-Ball' calibration of a 5-axis machining center, Annals of the CIRP 55 (1) (2006) 531-534.

[10] S. Ibaraki, C. Oyama, H. Otsubo, Construction of an error map of rotary axes on a five-axis machining center by static R-test, International Journal of Machine Tools and Manufacture 51 (2011) 190-200.

[11] S. H. H. Zargarbashi, J. R. R. Mayer, Single setup estimation of a five axis machine tool eight link errors by programmed end point constraint and on the fly measurement with Capball sensors, International Journal of Machine Tools and Manufacture 49 (2009) 759-766.

[12] ISO/DIS 10791-6:2012 Test conditions for machining centres - Part 6: Accuracy of speeds and interpolations, 2012.

[13] C. Hong, S. Ibaraki, A. Matsubara, Influence of Position-dependent Geometric Errors of Rotary Axes on a Machining Test of Cone Frustum by Five-axis Machine Tools, Precision Engineering 35 (1) (2011) 1-11.

[14] C. Hong, S. Ibaraki, Graphical presentation of error motions of rotary axes on a five-axis machine tool by static R-test with separating the influence of squareness errors of linear axes, International Journal of Machine Tools and Manufacture 59 (2012) 24-33.

[15] ISO 230-10:2011, Test code for machine tools - Part 10: Determination of measuring performance of probing systems of numerically controlled machine tools, 2010 . 
[16] US Patent, LS 7278222, Method for measuring a program-controlled machine tool, 2007.

[17] T. Erkan, J. R. R. Mayer, and Y. Dupont, Volumetric distortion assessment of a five-axis machine by probing a $3 \mathrm{D}$ reconfigurable uncalibrated master ball artefact, Precision Engineering 35 (2011) 116-125.

[18] T. Matsushita and T. Oki, Identification of Geometric Errors in Fiveaxis Controlled Machine Tool with Touch Trigger Probe, Proc. of the 2010 Spring JSPE Semiannual meeting (2010) 1105-1106 (in Japanese).

[19] S. Ibaraki, T. Iritani, T. Matsushita, Calibration of location errors of rotary axes on five-axis machine tools by on-the-machine measurement using a touch-trigger probe, International Journal of Machine Tools and Manufacture 58 (2012) 44-53.

[20] ISO 10360-3:2000, Geometrical Product Specifications (GPS) - Acceptance and reverification tests for coordinate measuring machines (CMM) - Part 3: CMMs with the axis of a rotary table as the fourth axis, (2000).

[21] ISO 230-1:2012, Test code for machine tools - Part 1: Geometric accuracy of machines operating under no-load or quasi-static conditions, 2012.

[22] I. Inasaki, K. Kishinami, S. Sakamoto, N. Sugimura, Y. Takeuchi, F. Tanaka, Shaper generation theory of machine tools - its basis and applications, Yokendo, Tokyo, 1997 (in Japanese).

[23] Renishaw, RMP600 high accuracy radio machine probe (Data sheet, H-5312-8200-06-A), http: //www.renishaw .com

[24] JCGM 100:2008, Evaluation of measurement data - Guide to the expression of uncertainty in measurement (GUM), 2008.

[25] B. Bringmann, J. Besuchet, L. Rohr, Systematic evaluation of calibration methods, CIRP Annals - Manufacturing Technology 57(1) (2008) $529-532$.

[26] B. Bringmann, W. Knapp, Machine tool calibration: Geometric test uncertainty depends on machine tool performance, Precision Engineering 33 (2009) 524-529. 
Table 1: Description and notation of position-dependent geometric errors associated with rotary axes for the machine configuration in Fig. 1. Correspondence to symbols depends on the setup of the machine tool coordinate system [21].

\begin{tabular}{l|l|l}
\hline Symbol $[22]$ & Symbol $[21]$ & Description \\
\hline$\alpha_{A Y}\left(A_{i}\right)$ & $E_{A A}$ & Angular positioning error of A-axis at $A_{i}$ \\
$\beta_{A Y}\left(A_{i}\right)$ & $E_{B A}$ & Parallelism error of A- to X-axis around Y-axis at $A_{i}$ \\
$\gamma_{A Y}\left(A_{i}\right)$ & $E_{C A}$ & Squareness error of A- to Y-axis at $A_{i}$ \\
$\delta x_{A Y}\left(A_{i}\right)$ & $E_{X 0 C}$ & C-axis position error in X-direction at $A_{i}$ \\
$\delta y_{A Y}\left(A_{i}\right)$ & $E_{Y A}$ & Radial error motion of A-axis in Y-direction at $A_{i}$ \\
$\delta z_{A Y}\left(A_{i}\right)$ & $E_{Z A}$ & Radial error motion of A-axis in Z-direction at $A_{i}$ \\
$\alpha_{C A}\left(A_{i}, C_{j}\right)$ & $E_{A C}-E_{A A}$ & Tilt error motion of C-axis around X-axis at $A_{i}$ and $C_{j}$ \\
$\beta_{C A}\left(A_{i}, C_{j}\right)$ & $E_{B C}-E_{B A}$ & Tilt error motion of C-axis around Y-axis at $A_{i}$ and $C_{j}$ \\
$\gamma_{C A}\left(A_{i}, C_{j}\right)$ & $E_{C C}-E_{C A}$ & Angular positioning error of C-axis \\
$\delta x_{C A}\left(A_{i}, C_{j}\right)$ & $E_{X C}$ & Radial error motion of C-axis in X-direction at $A_{i}$ and $C_{j}$ \\
$\delta y_{C A}\left(A_{i}, C_{j}\right)$ & $E_{Y C}-E_{Y A}$ & Radial error motion of C-axis in Y-direction at $A_{i}$ and $C_{j}$ \\
$\delta z_{C A}\left(A_{i}, C_{j}\right)$ & $E_{Z C}-E_{Z A}$ & Axial error motion of C-axis in Z-direction at $A_{i}$ and $C_{j}$ \\
\hline
\end{tabular}

Table 2: Machine tool specifications.

\begin{tabular}{l|l}
\hline Stroke & X:1250 mm, Y:660 mm, Z:540 mm, \\
& A: $+20^{\circ} \sim-110^{\circ}, \mathrm{C}: 360^{\circ}$. \\
\hline Table size & $\phi 500 \mathrm{~mm}$ \\
\hline Drives & X, Y, and Z: rotary servo motor with ballscrew, \\
& A and C: direct drive \\
\hline
\end{tabular}

Table 3: Touch-trigger probe and stylus specifications (RMP-600 by Renishaw [23]).

\begin{tabular}{l|l}
\hline Primary application & inspection probe for machining centers \\
\hline Sense directions & Omni-directional $\pm \mathrm{X}, \pm \mathrm{Y}, \pm \mathrm{Z}$. \\
\hline $\begin{array}{l}\text { Repeatability (in catalog; } \\
\text { max } 2 \sigma \text { value at stylus tip) }\end{array}$ & $0.35 \mu \mathrm{m}$ \\
\hline Signal transmission & $\begin{array}{l}\text { Frequency hopping spread } \\
\text { spectrum (FHSS) radio }\end{array}$ \\
\hline Probe sphere & $\phi 6 \mathrm{~mm}$ (ruby) \\
\hline Stylus length & $100 \mathrm{~mm}$ \\
\hline
\end{tabular}


Table 4: Contributors for identification uncertainties.

\begin{tabular}{l|l}
\hline Contributors & Standard uncertainty \\
\hline Probing & $0.7 \mu \mathrm{m}$ \\
- unidirectional repeatability & 0 \\
- directional pre-travel variation & \\
\hline Linear axes, X-axis & $1 \mu \mathrm{m} / \mathrm{mm}$ \\
- linear error, $E_{x x, l i n}$ & $2 \mu \mathrm{m}$ \\
- non-systematic error, $E_{x x, r a n d}$ & $2 \mu \mathrm{m}$ \\
- straightness error, $E_{y x, \text { rand }}, E_{z x, \text { rand }}$ & \\
Y-axis & $1 \mu \mathrm{m} / \mathrm{mm}$ \\
- linear error, $E_{y y, l i n}$ & $2 \mu \mathrm{m}$ \\
- non-systematic error, $E_{y y, r a n d}$ & $2 \mu \mathrm{m}$ \\
- straightness error, $E_{x y, r a n d}, E_{z y, r a n d}$ & $3 \mu \mathrm{m} / 100 \mathrm{~mm}$ \\
- squareness of Y- to X-axis, $S_{x y}$ & \\
Z-axis & $1 \mu \mathrm{m} / \mathrm{mm}$ \\
- linear error, $E_{z z, l i n}$ & $2 \mu \mathrm{m}$ \\
- non-systematic error, $E_{z z, r a n d}$ & $2 \mu \mathrm{m}$ \\
- straightness error, $E_{x z, r a n d}, E_{y z, \text { rand }}$ & $3 \mu \mathrm{m} / 100 \mathrm{~mm}$ \\
- squareness of Z- to X-axis, $S_{x z}$ & $3 \mu \mathrm{m} / 100 \mathrm{~mm}$ \\
- squareness of Z- to Y-axis, $S_{y z}$ & $10,10,120 \mu \mathrm{m}$ \\
\hline Each test piece & $10,10,10 \mu \mathrm{m} / 100 \mathrm{~mm}$ \\
- position error in X, Y, Z & $1 \mu \mathrm{m}$ \\
- orientation error around X, Y, Z & \\
- influence of surface roughness to probing &
\end{tabular}

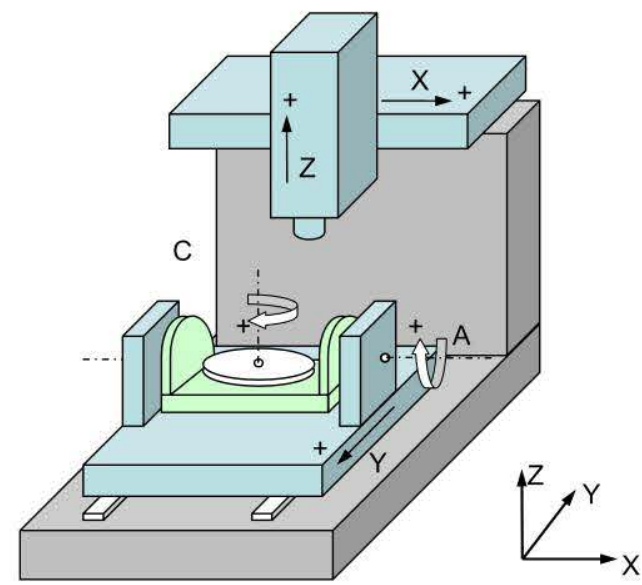

Figure 1: The configuration of the five-axis machine tool considered in this paper. 




(a) At $A_{i}=0^{\circ}$ and $C_{j}=0^{\circ}$. 


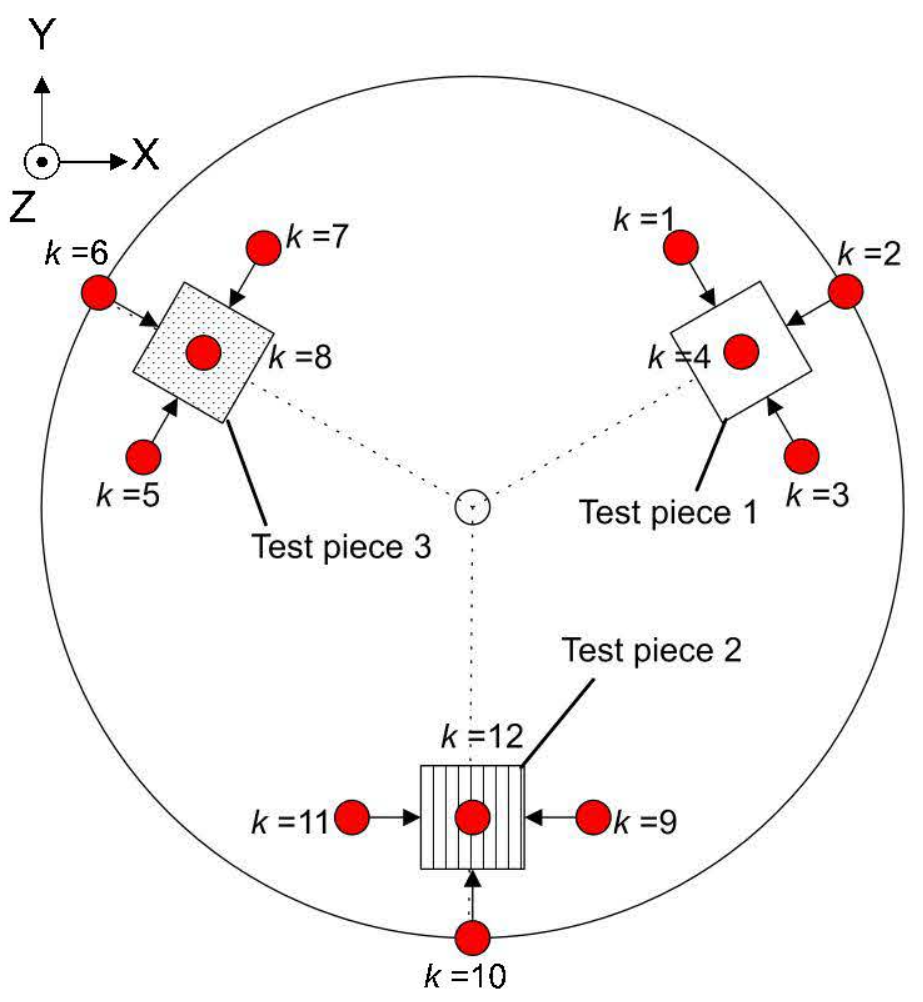

(b) At $A_{i}=0^{\circ}$ and $C_{j}=60^{\circ}$. 


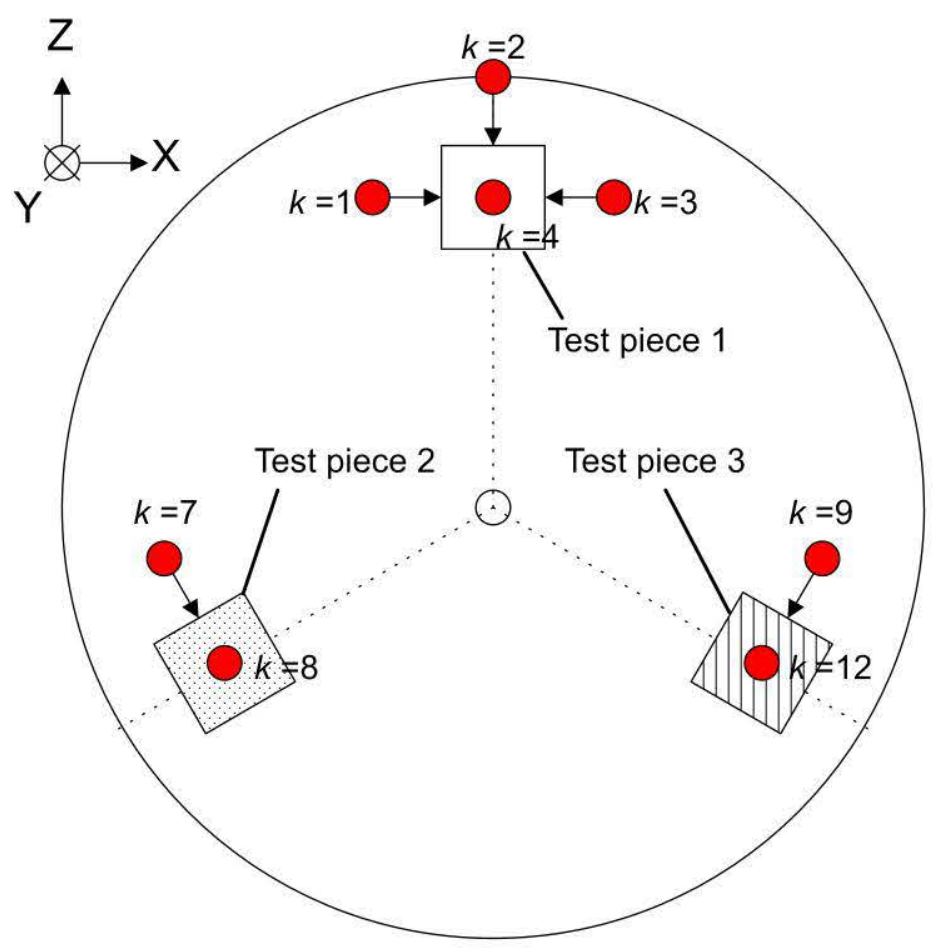

(c) At $A_{i}=-90^{\circ}$ and $C_{j}=0^{\circ}$.

Figure 2: Probed points at $\left(A_{i}, C_{j}\right)$. 


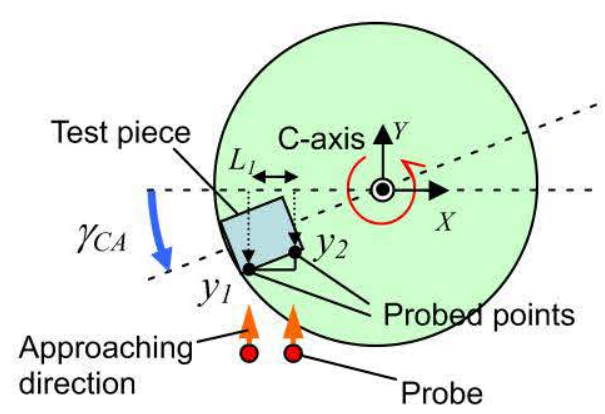

(a) With a single test piece.

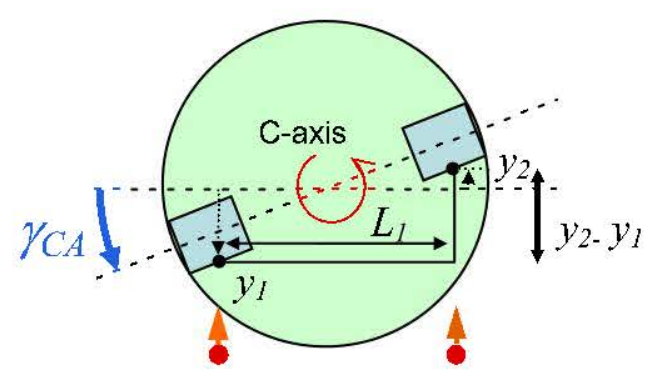

(b) With two test pieces.

Figure 3: Magnification of the influence of angular positioning error of $\mathrm{C}$-axis $\left(\gamma_{C A}\left(C_{j}, A_{i}\right)\right)$ by using two test pieces. 


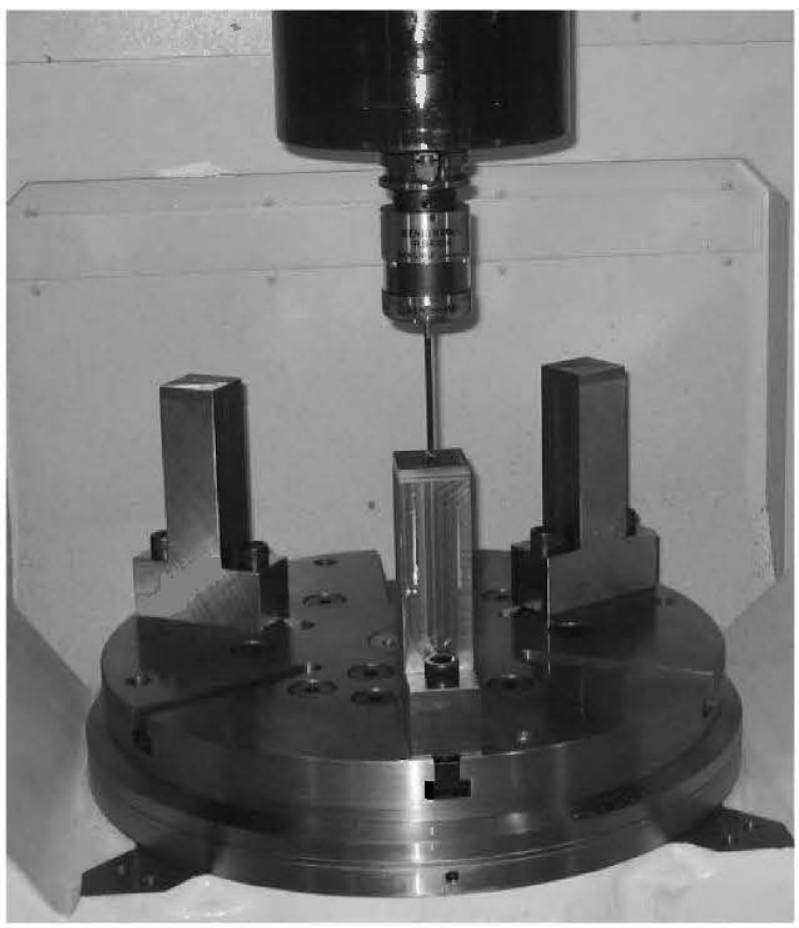

(a) At $A_{i}=0^{\circ}$ and $C_{j}=180^{\circ}$.

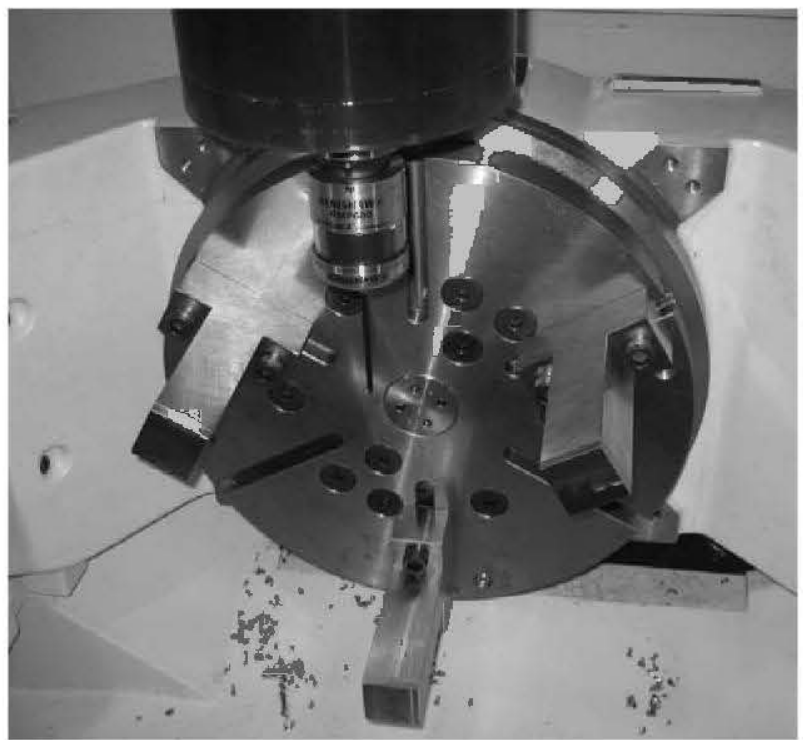

(b) At $A_{i}=-90^{\circ}$ and $C_{j}=180^{\circ}$.

24

Figure 4: Experimental setup. 


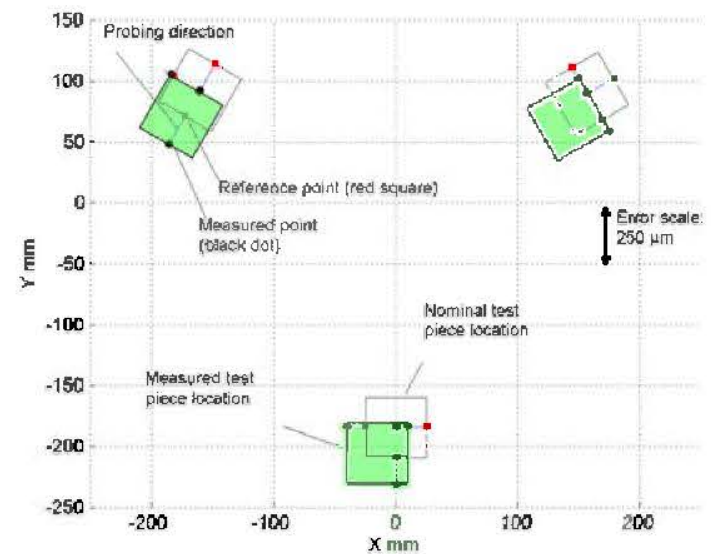

(a-1) At $A_{i}=0^{\circ}$ and $C_{j}=180^{\circ}$ (projected onto XY plane).

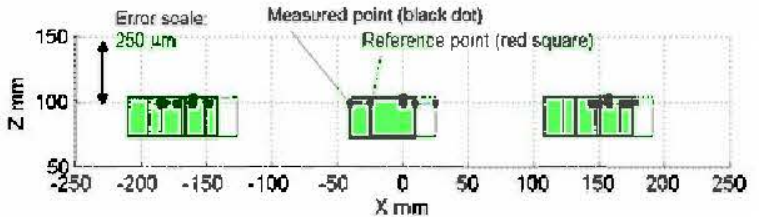

(a-2) At $A_{i}=0^{\circ}$ and $C_{j}=180^{\circ}$ (projected onto XZ plane). 


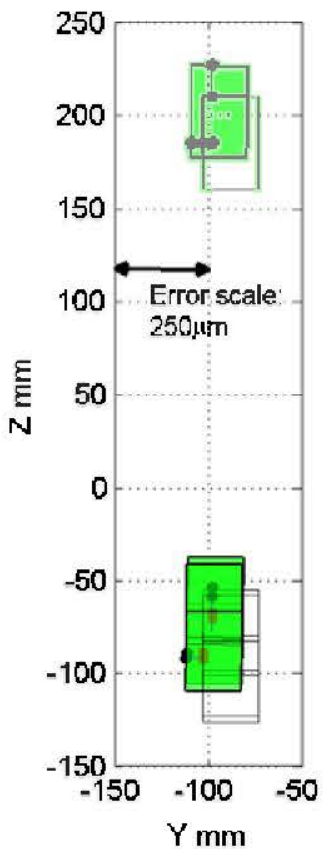

(b-1) At $A_{i}=-90^{\circ}$ and $C_{j}=0^{\circ}$ (projected onto $\mathrm{YZ}$ plane). 


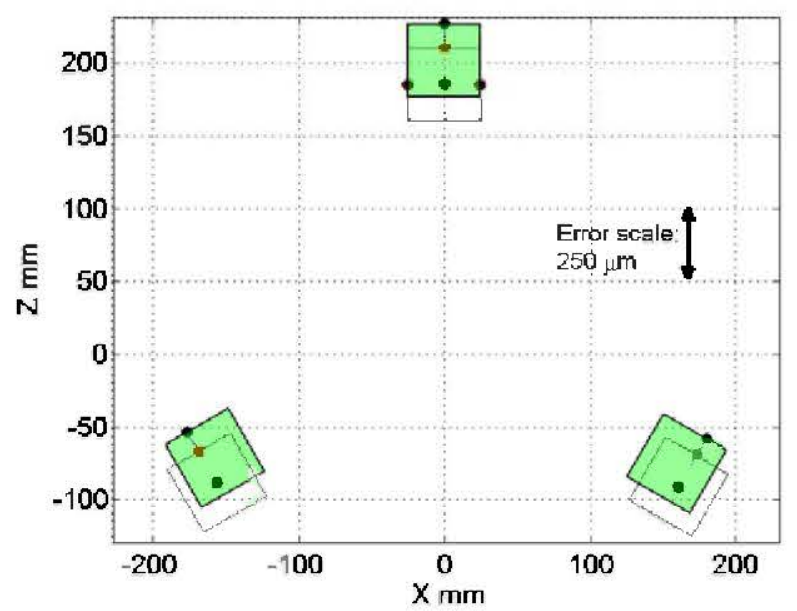

(b-2) At $A_{i}=-90^{\circ}$ and $C_{j}=0^{\circ}$ (projected onto XZ plane).

Figure 5: Probed point positions and estimated test piece positions and orientations (at (a) $A_{i}=0^{\circ}, C_{j}=180^{\circ}$, and (b) $A_{i}=-90^{\circ}, C_{j}=0^{\circ}$, as examples. 


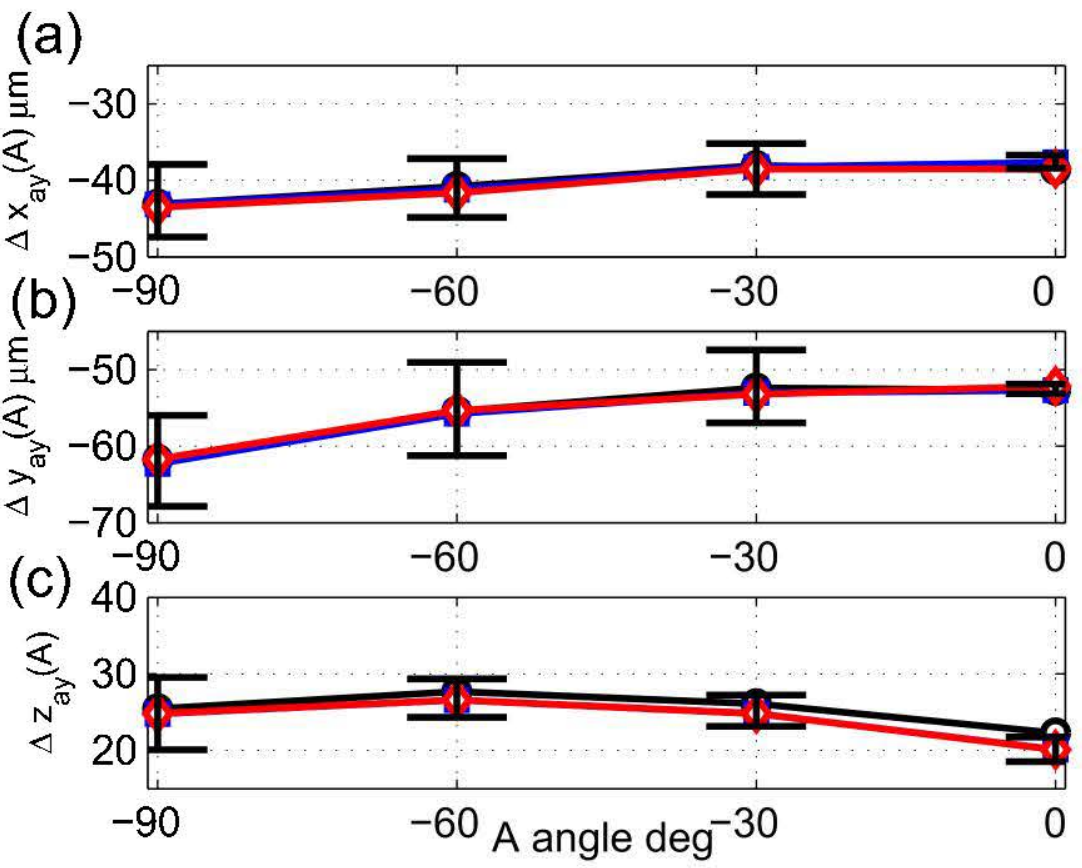

(d)
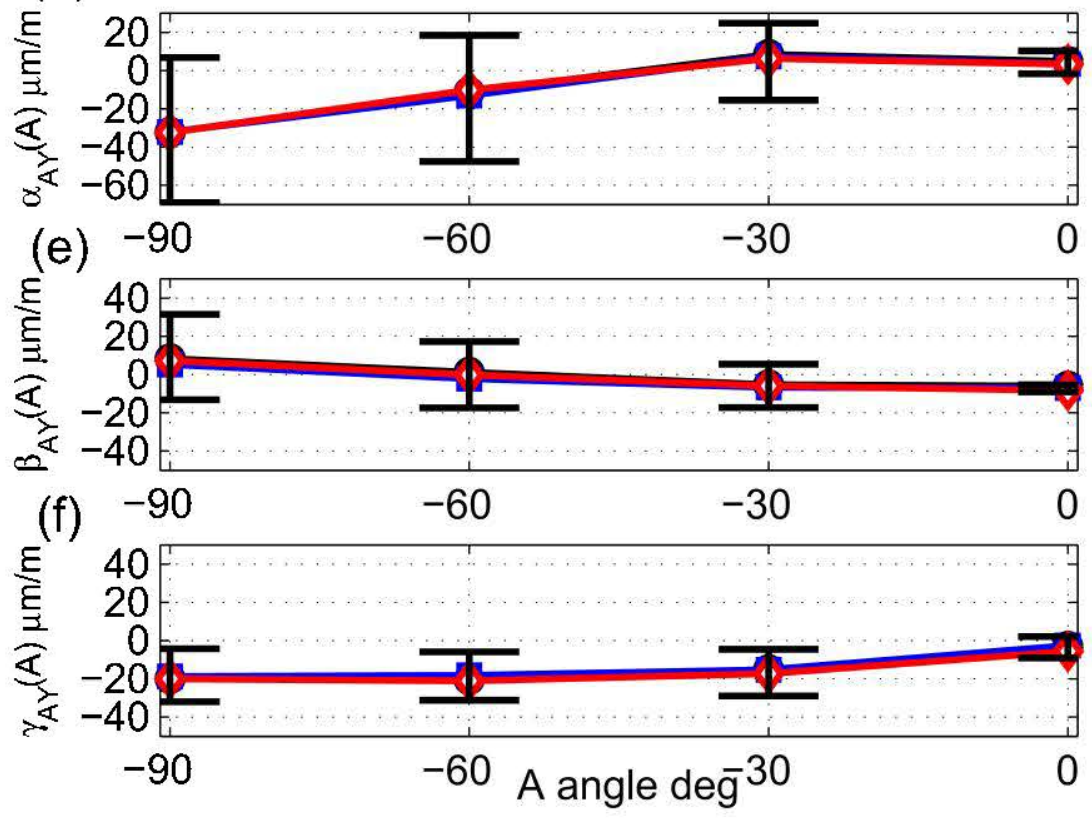

Figure 6: Estimated position-dependent geometric errors of A-axis. The present probing test was repeated three times, and the estimates at each test are all shown. Error bars represent the estimation uncertainty due to contributors shown in Table 4. (a) $\delta x_{A Y}\left(A_{i}\right)$, (b) $\delta y_{A Y}\left(A_{i}\right)$, (c) $28_{\Lambda Y}\left(A_{i}\right)$, (d) $\alpha_{A Y}\left(A_{i}\right)$, (e) $\beta_{A Y}\left(A_{i}\right)$, and (f) $\gamma_{A Y}\left(A_{i}\right)$. 

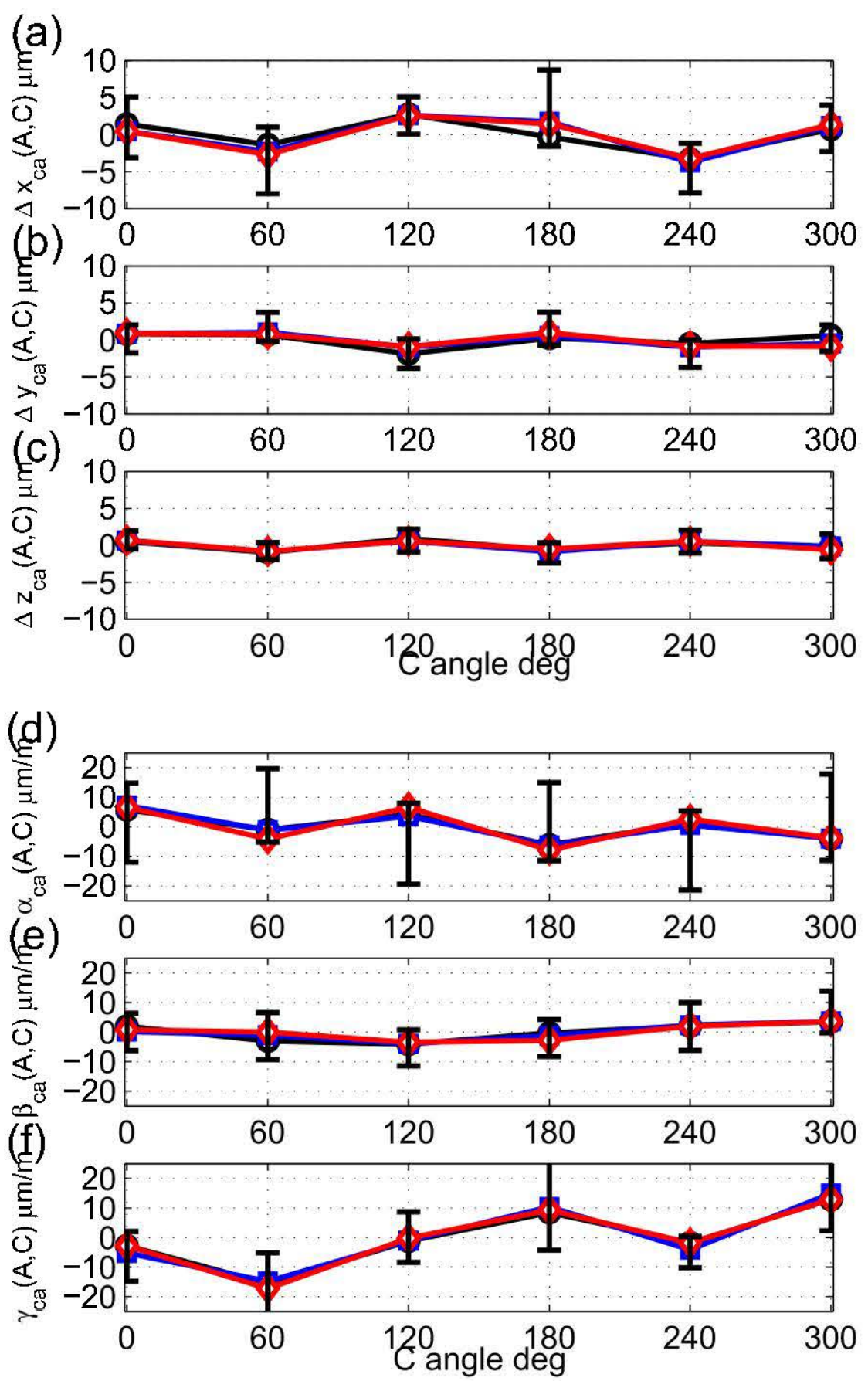

Figure 7: Estimated position-dependent geometric errors of C-axis at $A_{i}=-90^{\circ}$. The present probing test was repeated three times, and the estimates at each test are all shown. Error bars represent the estimation uncertainty due to contributors shown in Table 4. (a) $\left.\delta x_{C A}\left(-90^{\circ}, C_{j}\right), 29 \mathrm{~b}\right) \delta y_{C A}\left(-90^{\circ}, C_{j}\right)$, (c) $\delta z_{C A}\left(-90^{\circ}, C_{j}\right)$, (d) $\left.\alpha_{C A}\left(-90^{\circ}, C_{j}\right)\right)$, (e) $\beta_{C A}\left(-90^{\circ}, C_{j}\right)$, and (f) $\gamma_{C A}\left(-90^{\circ}, C_{j}\right)$. 